\title{
Los portales educativos como instrumentos innovadores para la formación en competencias informacionales de los estudiantes universitarios
}

\author{
Educational portals as innovative tools for teaching information skills to higher eduction students
}

\author{
María Pinto MolinA \\ Facultad de Comunicación y Documentación, Universidad de Granada, España \\ mpinto@ugr.es
}

\begin{abstract}
Resumen
En línea con la filosofía del Espacio Europeo de Educación Superior, el impacto de las tecnologías de la información y comunicación en la educación y los postulados del modelo socio-constructivista de aprendizaje de los estudiantes en el marco de la enseñanza superior, se reflexiona sobre el uso de los portales educativos como instrumentos innovadores para el aprendizaje de las competencias informacionales, tanto en el aula como fuera de ella. Partiendo de la experiencia de la autora en el desarrollo de varios portales dedicados a la formación en competencias informacionales -e-COMS, Alfin-EEES y Alfintra 2.0-, se exponen las principales fortalezas y debilidades y se avanzan algunas tendencias para los nuevos productos, útiles también para el aprendizaje a lo largo y ancho de la vida.
\end{abstract}

Palabras clave: Portales educativos. Alfabetización informacional. Competencias informacionales. Educación superior. e-COMS. Alfin-EEES. Alfintra 2.0.

\section{Introducción}

Las sociedades desarrolladas se ven sometidas a un proceso acelerado de cambio debido al impacto de las tecnologías de la información y comunicación, y la tendencia a la globalización. Las universidades están asumiendo las nuevas tecnologías como instrumentos para mejorar la calidad de los servicios educativos y adoptan una cultura en la que el conocimiento se sitúa en el centro de los procesos productivos y de la competitividad económica. Así se sitúan en la encrucijada de la denominada Sociedad de la Información y del Conocimiento, en la que una parte significativa de la actividad humana y la generación de riqueza proceden de la producción, procesamiento, difusión y utilización del conocimiento. En este sentido, el cambio más grande de nuestra sociedad está en el conocimiento, en su forma y contenido, en su signifi-

\begin{abstract}
A reflection on the role of educational portals as innovative tools to promote the development of information skills, in both online and face-to-face contexts. Their role is framed inside three broader contexts: the European Higher Education Space, the postulates of the socio-constructivist model of learning in higher education and the impact of the new information and communication technologies in education. Building on the author's experience in the development of three projects - e-COMS, Alfin-EEES y Alfintra 2.0-, their main strengths and weaknesses are analysed and some of the more important trends in course are discussed, which are of especial importance considereing the growing interest in promote long-life learning.
\end{abstract}

Keywords: Educational portals. Information literacy. Information skills. Higher education. e-COMS. AlfinEEES. Alfintra 2.0.

cado, en su responsabilidad y en lo que significa ser una persona competente (Drucker, 1999).

La educación superior ha experimentado diversas revoluciones en los últimos años, con transformaciones sin precedentes como, entre otras, la movilidad social del estudiante, las nuevas pautas de financiación de la enseñanza superior, la diversificación de los sistemas de enseñanza, la competitividad entre las universidades, la presencia de las TIC, la aparición de una red internacional de conocimientos y la disminución global de los niveles académicos. Las nuevas tecnologías de la información y la comunicación han creado un medio universal de contacto instantáneo y comunicación científica simplificada. También ha habido una importante concentración de importantes recursos de información en manos de las universidades más fuertes (Altbach, Reisberg y Rumbley, 2009). 
En general, la universidad está respondiendo al reto de la nueva configuración social basada en las TIC, empleando modelos de enseñanza a distancia como el e-learning y otras innovaciones centradas en el uso de las redes sociales con fines académicos. Las TIC han transformado el escenario de la enseñanza a distancia, al permitir un aumento del numero y tipos de prestatarios, nueva organización de los contenidos, nuevos modos de impartir la enseñanza e innovaciones pedagógicas. También ha cambiado en los últimos años el perfil de los estudiantes que se incorporan a la universidad: poseen un conocimiento cercano de las tecnologías, emplean nuevos hábitos de comunicación, se interesan por nuevas formas de enseñanza y autoaprendizaje en línea, demandan simplicidad en la transmisión de conocimientos, se preocupan por ser competitivos... Sin embargo, pese a estos cambios, se observa que tienen serias dificultades para relacionarse con la abundancia de información, y por tanto necesitan una mayor formación en su manejo, sobre todo en la adquisición de habilidades y competencias de análisis, síntesis, evaluación, difusión y uso de la información. Nos situamos en el escenario de la alfabetización informacional (ALFIN), entendida como el conjunto de aptitudes referidas al uso y dominio de la información en cualquiera de las formas en que se presente, así como de las tecnologías que dan acceso a esa información.

Los actuales sistemas de educación superior tienen que adaptarse a estos cambios, apostando fundamentalmente por la formación en competencias de los estudiantes, que potencie su autonomía y espíritu reflexivo para ejercer con éxito en el mercado de trabajo, renovando los métodos de enseñanza, y fomentando la capacidad de aprendizaje a lo largo de la vida.

La Unión Europea estableció en el año 2003 como uno de los objetivos fundamentales la promoción de una sociedad basada en el conocimiento, donde las universidades desempeñen un papel importante. Su doble función de centros de investigación y enseñanza, las convierte en piezas clave de la economía y la sociedad del conocimiento (Comisión Europea, 20022003). El papel que han de desempeñar como productoras, transmisoras y difusoras del saber científico y del saber hacer, les otorga un protagonismo en la formación y el desarrollo de los ciudadanos del siglo XXI, asumiendo una gran responsabilidad para afrontar los cambios que ha supuesto el establecimiento del Espacio Europeo de Educación Superior (EEES). Se trata de un espacio educativo común que permita la movilidad de estudiantes, docentes y PAS, y un sistema de estudios flexible, basado en una estructura de títulos y créditos comparable, y en el uso de metodologías activas e innovadoras.

\section{Innovación y formación por competencias}

A partir del Año Europeo de la Creatividad y la Innovación (2009), la Unión Europea está promocionando estos conceptos desde una perspectiva instrumental, como motores de crecimiento económico y de desarrollo social en las universidades. La innovación, desde un enfoque amplio y holístico -educativa, investigadora, tecnológica..., centrada primordialmente en la persona- es un pilar esencial para el desarrollo, la promoción, el impacto y la transferencia de conocimiento en las universidades. Innovar es aplicar nuevas ideas y pensamientos creativos realizados por mentes desafiantes y visionarias, que consiguen introducir variaciones como resultados de procesos de evaluación y ajuste, cambiando la forma de competir dentro de la organización. La innovación educativa se ha convertido en un requerimiento para la mejora del ciclo enseñanza-aprendizaje, representando una oportunidad de cambio estratégico para poder hacer frente a los retos de la sociedad de la información y del conocimiento. Desde una perspectiva innovadora, concebimos el aprendizaje como un continuum que tiene distintos estadios y tiempos en el contexto de la vida de una persona, que es capaz de aprender de los recursos que la rodean, haciendo de la vida un permanente estado de aprendizaje y adaptación que trasciende cualquier plan de estudios (Sharples, Taylor, Vavoula, 2005).

La creación de una Europa basada en el conocimiento representa para las universidades una fuente de oportunidades y también considerables desafíos. De una educación centrada básicamente en la docencia, en la que el profesor actúa como principal agente de enseñanza y el estudiante es un receptor de conocimientos, se está evolucionando hacia un modelo basado en el aprendizaje, en el cual el estudiante se convierte en protagonista activo, adquiriendo una serie de recursos que le permiten continuar aprendiendo a lo largo de su vida. El proceso de mejora que lo posibilite ha de ser interactivo y se sustenta en tres principios básicos:

- Mayor implicación y autonomía del estudiante.

- Utilización de metodologías más activas: casos prácticos, trabajo en equipo, tutorías, seminarios, tecnologías multimedia, etc. 
- Papel del profesor como agente creador de entornos de aprendizaje que estimulen al estudiante.

Este nuevo modelo educativo propone un aprendizaje basado en una serie de competencias que reflejan la capacidad del estudiante para poner en práctica de manera integrada habilidades, conocimientos y valores (es decir, competencias) para enfrentarse y poder resolver problemas y situaciones. Pero una competencia es algo más que conocimiento, habilidades y valores. Es la capacidad de hacer frente a demandas complejas, poniendo en funcionamiento nuestros recursos psicosociales (incluyendo habilidades y actitudes) en un contexto determinado (OECD, 2005).

Por tanto, en este marco de actuación el papel de las competencias genéricas y transversales es significativo, como también de aquellas otras que van más allá de las establecidas en el curriculo formal, y están relacionadas con la calidad de uso de la información y del conocimiento. Sabemos que las competencias informacionales y digitales son fundamentales en la formación de los estudiantes del siglo XXI, aunque paradójicamente en muchas ocasiones este aprendizaje no se materialice formalmente ni se visibilice en los actuales planes de estudio. A veces, el aprendizaje de estas competencias se realiza de manera informal, sin que haya enseñanza explícita, a través de la colaboración e interactuación de los estudiantes, siendo más bien un aprendizaje social. El desafío es que ambas competencias -informacionales y digitalesrequieren ser estimuladas de manera formal, ser planificadas y contextualizadas en la práctica, en definitiva estar visibles en los planes de estudio de los títulos de grado, y por tanto en el curriculum del estudiante. Así se cumpliría con las desideratas de los documentos programáticos que ensalzan la importancia de adquirir estas competencias en el marco de la enseñanza superior.

Desde el punto de vista de la programación de una formación especializada dentro de una perspectiva de educación integral, conviene diferenciar entre dos tipos de competencias (Pinto y Garcia, 2007):

- Básicas, genéricas o transversales, necesarias para todas las profesiones en mayor o menor medida. Se trata de competencias relacionadas con la lectura, la escritura, el cálculo, las tecnologías de la información y la cultura tecnológica, lenguas extranjeras, la comunicación, la resolución de problemas, el razonamiento, la capacidad de liderazgo, la creatividad, la motivación, el trabajo en equi- po y especialmente la capacidad de aprender... La adquisición de estas competencias permitirá al estudiante afrontar las competencias específicas de su área de conocimiento. Son transferibles a una gran variedad de funciones y tareas, y capacitan y habilitan al estudiante para integrarse con éxito en la vida laboral y social.

- Específicas, propias de cada perfil profesional.

\section{La nuclearidad de las competencias informacionales}

La Alfabetización Informacional y el desarrollo de competencias informacionales se relacionan con los enfoques constructivistas del aprendizaje. En ellos el estudiante hace un aprendizaje significativo, que parte de sus conocimientos previos, y activo, reflexivo e intencional en la realización de sus tareas (Johnson, 2007). Se preconizan métodos activos de aprendizaje, basados en la resolución de problemas y el estudio de casos, y se fomenta el desarrollo de habilidades cognitivas, haciendo que el estudiante sistematice su trabajo, mejore la comprensión e interpretación textual y potencie el pensamiento crítico y creativo. También los profesores, tutores y bibliotecarios necesitan, para contribuir a este proceso de aprendizaje, adquirir mejores competencias informacionalespedagógicas-didácticas (Cvetkovic y Lackie, 2009) y apoyarse en los distintos instrumentos tecnológicos que facilitan estos procesos de enseñanza-aprendizaje, como son las plataformas e-learning y la Web 2.0 (Levy y Roberts, 2005; York y Vance, 2009; Xiao, 2010; Konieczny, 2010).

En el actual contexto de la sociedad de la información y del aprendizaje, el conocimiento y dominio de los procesos asociados a la búsqueda, la evaluación, el procesamiento, la comunicación y difusión de la información, son competencias nucleares para incrementar la eficacia del proceso de enseñanza-aprendizaje. La alfabetización es un concepto amplio, complejo, multidimensional y multifuncional, que acoge tanto la alfabetización básica (leer, interpretar y escribir) como la alfabetización visual, multimedia, informacional, digital, intercultural...La alfabetización informacional supone el dominio de un conjunto de conocimientos, habilidades y actitudes que capacita al estudiante para saber cuándo necesita información y cómo ha de buscarla, evaluarla, procesarla, gestionarla y utilizarla de forma ética y eficaz en un proyecto determinado (ALA, 1989). 
El estudiante universitario que es competente en términos informacionales:

- Adquiere y mejora sus competencias personales para aprender a aprender.

- Es autónomo en el aprendizaje y en el desarrollo de trabajos académicos.

- Es capaz de conocer la terminología de su especialidad.

- Sabe consultar y utilizar fuentes electrónicas de información, tanto revistas como bases de datos...

- Sabe emplear adecuadamente los recursos de información, valorando su calidad tanto formal como de contenido.

- Adquiere la competencia de análisis.

- Aprende a esquematizar y resumir información.

- Mejora su capacidad de organización de la información

- Aprende a utilizar las TIC con fines académicos.

- Sabe evitar el plagio en los trabajos académicos.

- Adquiere habilidades para mejorar la comunicación oral y escrita.

La importancia de la adquisición y aprendizaje de las competencias informacionales por parte de los estudiantes se evidencia tanto en los libros blanco de las titulaciones universitarias (Aneca, 2004) como en el RD 1393/2007 que articula la ordenación de las enseñanzas universitarias oficiales. De hecho entre los objetivos de los nuevos títulos de Grado se garantiza como mínimo que los estudiantes adquieran, entre otras las siguientes competencias básicas (recogemos aquellas relacionadas con la información):

[...] que los estudiantes tengan la capacidad de reunir e interpretar datos relevantes... para emitir juicios que incluyan una reflexión sobre temas relevantes de índole social, científica o ética.

[...] que los estudiantes puedan transmitir información, ideas, problemas y soluciones a un público tanto especializado como no especializado.

[...] que los estudiantes hayan desarrollado aqueIlas habilidades de aprendizaje necesarias para emprender estudios posteriores con un alto grado de autonomía".

En síntesis, aunque las competencias informacionales son importantes para los títulos de Grado, como recoge el texto legal, no se ha planificado su integración y articulación en los nuevos planes de estudio. Su aplicabilidad ha quedado relegada a criterio de cada universidad, observándose un divorcio entre la desiderata oficial (formación por competencias) y la realidad académica (formación convencional en proceso de cambio).

\section{El aprendizaje de las competencias informacionales a través de los portales de contenido}

Los portales de contenido son espacios virtuales que ofrecen múltiples servicios a los miembros de la comunidad universitaria. Constituyen un nodo integrado para el acceso comprensivo y útil de la información y además de contenidos ofrecen información, instrumentos para la búsqueda de información, recursos didácticos, materiales docentes interactivos, herramientas para la comunicación interpersonal, formación y asesoramiento (Eisler, 2000; Jafari, 2003). Constituyen un buen apoyo para la formación de los estudiantes universitarios en competencias genéricas y de información, por múltiples razones:

- Se adaptan bien a las nuevas exigencias del aprendizaje basado en TIC, que requieren de espacios formativos interactivos.

- Su enfoque basado en "aprender haciendo", permite adquirir las habilidades y competencias clave tanto durante sus estudios universitarios como a lo largo de la vida.

- Se apoyan en el modelo de aprendizaje significativo, que favorece el autoaprendizaje organizado y constructivo.

- Contribuyen a la formación del profesorado, ofreciéndole recursos, propuestas de actividades y recomendaciones.

Además, existen argumentos técnicos que refuerzan la utilidad de los portales en el proceso de enseñanza-aprendizaje en el marco del nuevo modelo educativo:

- Su orientación pedagógica: Dirigida al aprendizaje significativo, especialmente a aprender haciendo, proporcionan material didáctico, actividades y recursos innovadores dinámicos que motivan al estudiante, fortaleciendo su autonomía.

- La accesibilidad: Emplean recursos abiertos, gratuitos y accesibles, que permiten rapidez de descarga y consulta.

- La funcionalidad: En general, adoptan una interfaz intuitiva, con páginas poco extensas que utilizan un lenguaje claro y comprensible. 
- El contenido: Debe ser contenido abierto, pertinente, de calidad, interoperable y reusable. La información estará sistematizada, actualizada y estructurada, con encabezamientos claros y descriptivos e incluirá variedad de ejercicios como simulaciones, ejemplificaciones, crucigramas...

- La comunicación: Usan canales ágiles de comunicación para compartir ideas y materiales, debatir temas, consultar dudas (foro, chat, correo electrónico).

En general las funciones principales de los portales de contenido se concretan en las siguientes dimensiones:

- Ofrecen información en línea fiable de todo tipo a los miembros de la comunidad universitaria.

- Proporcionan recursos didácticos y educativos de todo tipo, gratuitos y utilizables directamente desde internet (materiales didácticos en línea) o desde los ordenadores (tras "bajar" una copia desde el portal).

- Contribuyen a la formación del estudiantado, ofreciendo una importante selección de contenidos, esquemas, mapas, dossieres y actividades, facilitando un entorno de colaboración amigable.

- Apoyan la labor del profesorado, ofreciendo estrategias y acciones para el diseño de materiales didácticos, experiencias e iniciativas similares, recursos comentados y evaluados...

- Permiten la integración en plataformas de aprendizaje virtual, tipo WebCT, Moodle...

- Ofrecen canales de comunicación (foros, chats, listas de distribución) entre los miembros de la comunidad universitaria, en los que se comparten ideas y materiales, se debaten temas, se consultan dudas...

- Proporcionan instrumentos para la comunicación: correo electrónico, chats, foros, espacios para alojar páginas web...

- Ofrecen garantías de accesibilidad y usabilidad de los contenidos.

\section{Algunas iniciativas}

De todos portales de contenido que hemos desarrollado desde el curso 2003-2004 para el aprendizaje de las competencias informacionales, nos centraremos en estos tres, especialmente por su uso e impacto en la comunidad universitaria
- e-COMS, portal de corte documental, centrado en las principales competencias informacionales.

- Alfin-EEES, portal que aborda las competencias genéricas y de información.

- ALFINTRA 2.0, portal orientado a la formación en competencias informacionales en el área de Traducción e Interpretación.

\subsection{El portal e-COMS}

El portal e-COMS -disponible en www.maria pinto.es/e-coms/- ha sido pionero en el ámbito de la información y documentación y fue concebido para potenciar el aprendizaje del estudiante universitario en lo referente a la gestión, evaluación y organización de los contenidos electrónicos (Pinto, 2004). Su amplia utilización por profesionales, docentes y estudiantes de distintos ámbitos geográficos nacionales e internacionales, ha hecho que sea un portal formativo e informativo de referencia para los estudiantes universitarios de biblioteconomía y documentación, de educación, psicopedagogía, ... al afrontar estos dos objetivos clave: por un lado, entrenar al estudiante en el conocimiento, habilidades y competencias para el análisis, organización de la información, evaluación de la calidad, iniciación a la investigación..., apostando por el desarrollo de competencias tecnológicas e informacionales; y por otro, ofrecer nuevos métodos docentes y estrategias de aprendizaje que contribuyan a la autonomía del estudiante y potencien su capacidad para resolver problemas.

El portal ofrece información y formación para los estudiantes en las principales competencias relacionadas con el ciclo de vida y producción de la información:

- Se trabaja la competencia de necesidad de información. Se le enseña al estudiante cómo definir y articular una necesidad de información con pensamiento analítico y creativo, a contextualizarla, a ordenar sus ideas con claridad, a identificar el problema de información, a planificar el tiempo que dedicará a la búsqueda, a definir los criterios y estrategias adecuadas, empleando las herramientas documentales necesarias.

- Se incide en la competencia de iniciación a la investigación, haciendo que los estudiantes entiendan los principios del pensamiento científico, los procesos de creación e innovación y los métodos y técnicas de investigación como instrumentos de apoyo para el desarrollo de sus trabajos. Es necesario que comprendan el carácter dinámico de la inves- 
tigación y su alcance para el desarrollo de proyectos, trabajos e informes.

- Se aborda la competencia relacionada con los contenidos electrónicos, especialmente cómo crearlos, modificarlos, gestionarlos, referenciarlos, transferirlos y utilizarlos.

- Se incide en las habilidades de organización, análisis, resumen y representación de la información.

- Se profundiza en la competencia de búsqueda y recuperación de información, y se ofrece información sistematizada sobre el manejo de diferentes métodos, técnicas y herramientas para garantizar una recuperación de calidad: bases de datos, Internet, tesauros, ontologías, mapas... El estudiante competente obtiene la información que necesita a través de los métodos adecuados, identifica las fuentes de información impresa y electrónicas posibles, utiliza las diferentes herramientas de búsqueda y accede adecuadamente a los recursos.
- También se hace hincapié en cómo evaluar la información, teniendo presente indicadores como su autenticidad, corrección, valor y sesgo. La facilidad y libertad con que pueden publicarse contenidos en Internet hace necesario que se adopten criterios para filtrar los contenidos electrónicos y poder discernir la veracidad, fiabilidad y en definitiva, la calidad de la información que este medio proporciona. El estudiante encuentra pistas para definir cuál es la información útil y relevante para resolver su problema de información y cómo aplicar criterios para evaluarla.

- Por último, se trabajan las competencias de comunicación y difusión de la información y se profundiza en la edición electrónica.

Entre los servicios que ofrece el portal se encuentran: un tutorial para el manejo del mismo; una selección de recursos electrónicos, buscadores, diccionarios y software; un glosario y FAQs; un foro; y un cuestionario para recoger la opinión de los usuarios y mejorar aquellos aspectos necesarios.
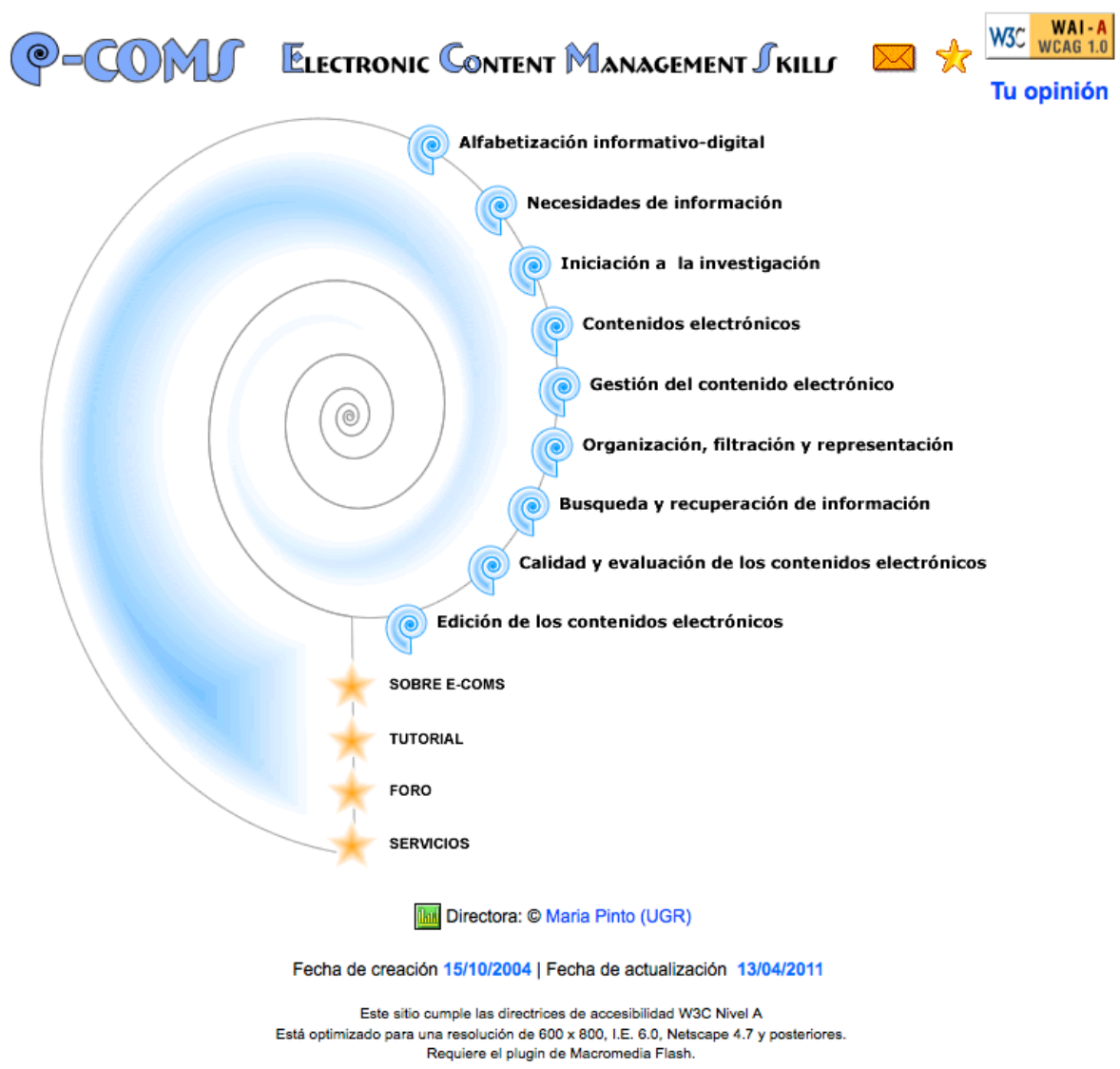

Figura 1. Página principal del portal e-Coms 
Tomando como referencia la frecuencia de consultas de una vez a la semana, las categorías más utilizadas por los estudiantes son las siguientes: calidad y evaluación de la información, búsqueda y recuperación, gestión de contenidos electrónicos, organización, representación y filtración, necesidades de información, edición de la información, contenidos electrónicos, alfabetización informacional e iniciación a la investigación. Es decir, ocupan las primeras posiciones aquellas competencias informacionales relacionadas con la evaluación, búsqueda y procesamiento de la información, todas ellas necesarias para el aprendizaje autónomo en el actual entorno electrónico.

\subsection{El portal AIFin-EEES}

El portal AIFin-EEES es el acrónimo de Alfabetización Informacional en el Espacio Europeo de Educación Superior, que se apoya en la experiencia previa adquirida en el desarrollo del portal e-COMS. Está disponible en http://www. mariapinto.es/alfineees/AlfinEEES.htm. Es un portal de formación e-learning sobre competencias genéricas y de información para los estudios superiores y ofrece formación en estas seis competencias (García y Pinto, 2010):
- Aprender a aprender: Se aborda cómo aprender a aprender, a ser autónomos y responsables de nuestro propio proceso de aprendizaje.

- Aprender a buscar y evaluar información.

- Aprender a analizar, sintetizar y comunicar: cómo leer mejor, cómo segmentar la información con el fin de reorganizarla, posteriormente, utilizando las técnicas de esquematización, representación gráfica y el resumen. Se aborda también cómo comunicar los nuevos conocimientos mediante la escritura y otros sistemas gráficos.

- Aprender a generar conocimiento: Se estudian los procesos de creación e innovación, técnicas de organización de proyectos, etc., con el fin de capacitar al estudiante en la creación de conocimiento.

- Aprender a trabajar con los demás: Se plantean los fundamentos éticos de la convivencia y del trabajo en equipo.

- Utilizar la tecnología para aprender: Se centra en los sistemas operativos, aplicaciones ofimáticas, herramientas de comunicación y entornos de e-learning más utilizados en el aprendizaje.

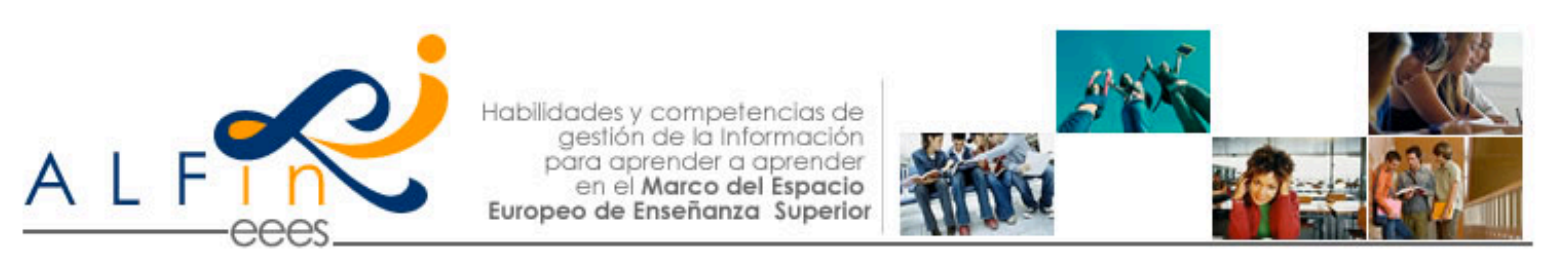

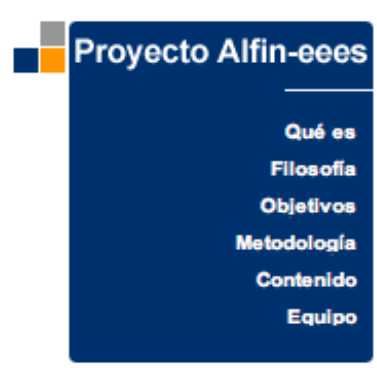

Competencias

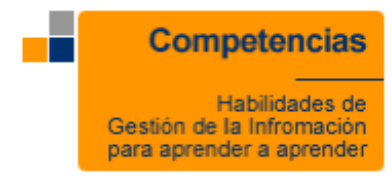

Alfin-EEES es una iniciativa piloto sobre la propuesta de contenidos de las principales COMPETENCIAS GENÉRICAS relacionadas con la alfabetización en información, validas para cualquier estudiante universitario que necesite buscar, gestionar, organizar y evaluar la información recogida en fuentes muy diversas. Algunos estudios cualificados norteamericanos han demostrado que la alfabetización en información contribuye a la mejora de los resultados de aprendizaje y ayuda a producir titulados capacitados para el aprendizaje a lo largo de la vida.

Alfin-EEES sirve para potenciar dicha alfabetización en información de quienes lo consultan, multiplicando las oportunidades de aprendizaje electrónico autodirigido y fomentando valores como la innovación, la creatividad.

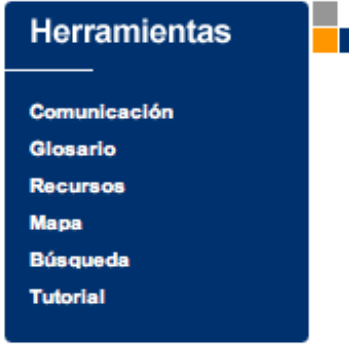

\section{(C) Alfin EEES, $2010 \quad$ Maria Pinto-UGR P}

Figura 2. Página principal del portal Alfin-EEES 
Cada competencia se despliega en subcompetencias, y éstas a su vez en temas, que incluyen la descripción conceptual, los procedimientos o el cómo aprenderla, esquemas, mapas, actividades y ejemplificaciones, además de un seleccionado dossier de recursos electrónicos (tutoriales, software, portales educativos, campus virtuales....) y un conjunto de recomendaciones de interés para estudiantes y profesores.

Las herramientas de soporte son de diferentes tipos: las herramientas de búsqueda de información, el mapa del sitio y el motor de búsqueda; ayuda para utilizar el sitio; lista de apoyo al estudio de materiales, glosario; y para la comunicación, foros, correo electrónico y chat.

Por su parte, los conocimientos se dividen en declarativos y procedimentales. Por ello, se incluyen apartados diferentes para las facetas declarativas (saber decir) y procedimentales (saber hacer), que en el contexto de los materiales ofrecidos a los estudiantes se expresan simplemente con las etiquetas Qué y Cómo. El objetivo es tratar ambos aspectos a un mismo nivel y comunicar con claridad al estudiante la importancia de equilibrar ambos tipos de conocimiento. Por ello se decidió distinguirlos gráficamente y con claridad mediante diferentes tablas.

Los conocimientos se expresan tanto con palabras como mediante imágenes a través de los mapas conceptuales. Se trata de expresar la información en otro lenguaje, de cartografiar los conceptos y de proporcionar instrumentos para la argumentación visual.

El apartado de actividades contiene siempre un ejemplo resuelto y un elenco de actividades que se proponen al alumno para desarrollar la competencia, subcompetencia o aspecto específico tratado. Se intenta que los ejemplos y las actividades propuestas sean de carácter general o aborden diferentes áreas de conocimiento, para mostrar al estudiante modelos cercanos a su campo de experiencia, que pueda generalizar o concretar según sus necesidades. Al menos una de las actividades ya aparece resuelta.

Se ofrecen también recursos para que el estudiante pueda ampliar sus conocimientos, así como material complementario, en particular aplicaciones informáticas. También se aportan una serie de consejos, para profesores y alumnos, con la idea de ayudarles a evitar algunas de las dificultades más importantes que los autores han experimentado al tratar de abordar estos temas

\subsection{El portal ALFINTRA 2.0}

Finalmente, nos referimos al portal ALFINTRA 2.0 , cuyo diseño se basó tanto en el conocimiento previo de los participantes como en los resultados de investigaciones realizadas a los estudiantes, docentes y profesionales en el contexto de la documentación aplicada a la traducción (Pinto y Sales, 2007; Sales, 2008). El objetivo principal del portal es fortalecer la formación del estudiante de traducción e interpretación en alfabetización informacional, para que sea capaz de reconocer y acotar una necesidad de información, localizar esa información, evaluar las fuentes, utilizar la información de forma efectiva y saber integrarla en la base de conocimiento. Está disponible en www.mariapinto.es lalfintra/.

Los resultados de estas investigaciones evidencian algunas características de las necesidades y expectativas informacionales de los estudiantes de Traducción e Interpretación que se tuvieron en cuenta para definir el diseño del portal ALFINTRA.

El estudiante de primer ciclo necesita refuerzo en todas las competencias informacionales, pero en su percepción apunta esencialmente a la necesidad de conocimiento de fuentes; comienza a ser consciente de la necesidad de entender la búsqueda, recuperación y gestión de la información como parte de un proceso en el que lo esencial es la perspectiva crítica que aporte calidad al resultado final; y todavía no se muestra consciente de la importancia de crear y mantener una propia base de conocimientos.

Por su parte, el estudiante de segundo ciclo demuestra tener mayor conocimiento del abanico de fuentes de información y es claramente consciente de los elementos del proceso de información, aunque reconozca necesitar refuerzo para su logro. Con respecto a la base de conocimientos, se muestra consciente de su relevancia, pero todavía le falta reforzar la competencia estratégico-procedimental para optimizar todas sus tareas documentales.

En suma, a la luz del diagnóstico de necesidades y expectativas, se diseñaron los contenidos del portal en el marco de la alfabetización informacional, para atender tanto a la transversalidad como a la especialización. Se ofrece una plataforma 2.0 interactiva de aprendizaje que aporta conocimientos, habilidades, estrategias y valores para el uso y tratamiento de la información, tan necesaria en su futura actividad traductora. Las competencias abordadas se detallan en la figura 3. 

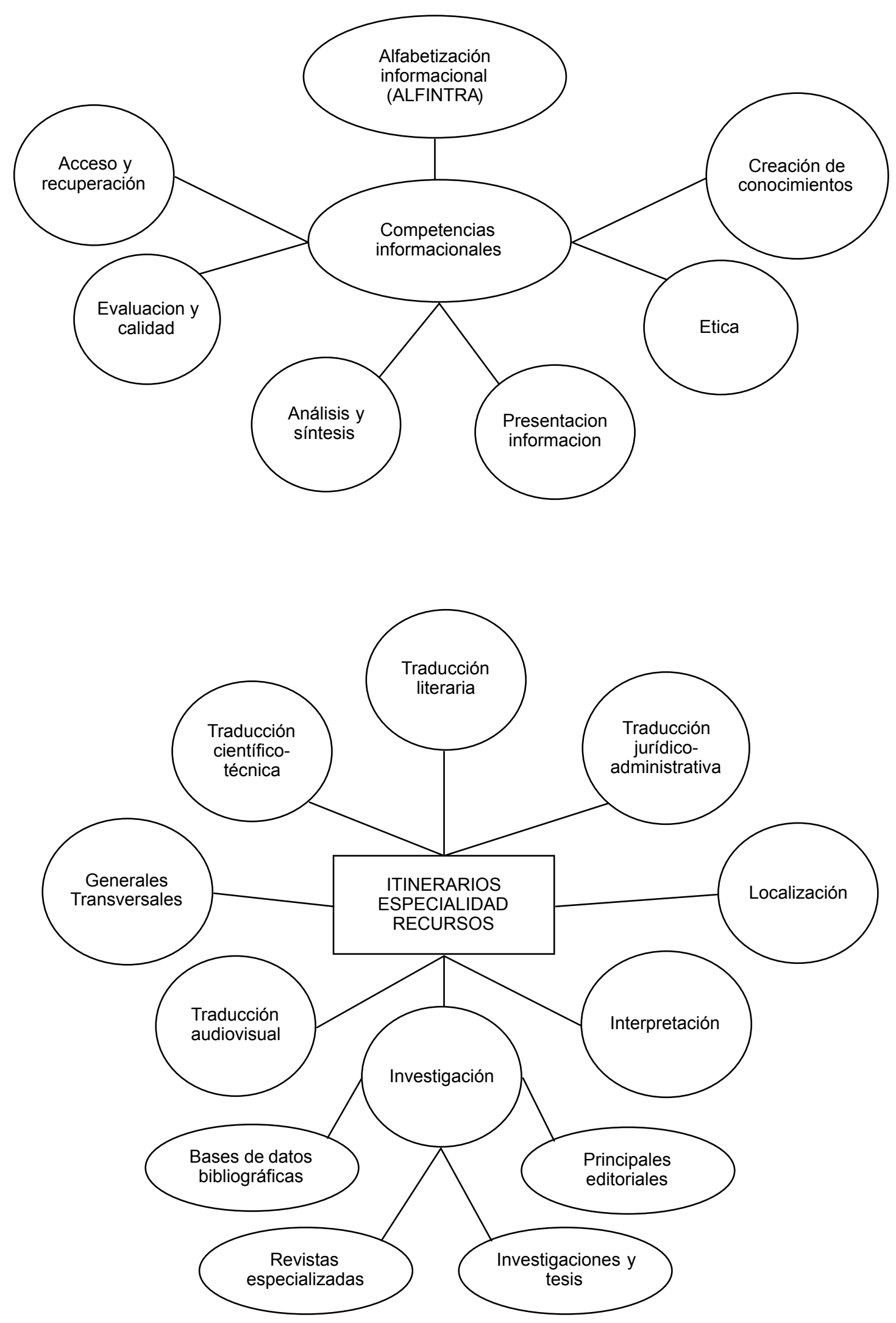

Figura 3. Categorias e itinerarios del portal ALFINTRA 2.0 


\section{Fortalezas y debilidades de los portales de contenidos}

De las iniciativas anteriormente relatadas, podemos inferir algunas fortalezas que a nuestro juicio aportan el uso de los portales de contenido educativos para el aprendizaje y la formación en alfabetización informacional.

Están dirigidos al aprendizaje de las principales competencias genéricas y de información, relacionadas con los aspectos importantes del ciclo de vida de la información y del conocimiento.

Abordan las competencias informacionales desde un enfoque integrador, poniendo énfasis en los siguientes aspectos: cognitivo, entrenando en las estrategias de análisis, síntesis, evaluación y procesamiento de la información; tecnológico, centrado en el aprendizaje de herramientas informáticas; documental, basado en el manejo de la información, reconociendo cuando existe una necesidad, saber buscar empleando estrategias para localizar y obtener información, saber emplear métodos básicos de investigación para la resolución de problemas, saber valorar y discriminar la información relevante para la toma de decisiones; y comunicativo, fomentando las habilidades para la comunicación, la participación y la difusión de información.

Son instrumentos de aprendizaje para el estudiante, especialmente para consolidar el entrenamiento en lo relativo a la búsqueda, evaluación, análisis y uso de la información, tan relevante en su formación y su futura vida profesional. Proporcionan recursos didácticos y educativos de todo tipo, gratuitos y utilizables directamente desde Internet (materiales didácticos online) o desde los ordenadores (tras "bajar" una copia desde el portal).

Son muy útiles para la formación a lo largo de la vida, ofreciendo una importante selección de contenidos, esquemas, mapas, dossieres y actividades, facilitando un entorno de colaboración amigable.

Apoyan la labor del profesorado, ofreciendo estrategias y acciones para el diseño de materiales didácticos de calidad, experiencias e iniciativas similares, recursos comentados y evaluados... Favorecen la innovación educativa, el trabajo en equipo, la compartición de conocimiento y la creación de redes docentes.

Permiten la integración en plataformas de aprendizaje virtual, tipo WebCT, Moodle. Son recursos de gran utilidad para la enseñanza elearning y blended learning, y se constituyen como ofertas educativas de actualidad que pue- den ayudar a promocionar el aprendizaje autónomo y el autoaprendizaje del estudiante.

Destaca el impacto de uso y la visibilidad que han tenido cualquiera de estos portales tanto a nivel local como global, al ser utilizados por estudiantes universitarios de toda índole académica y geografica, bibliotecarios, profesores, maestros, investigadores... Sirva de botón de muestra los datos de uso del portal Alfil-EEES (Garcia y Pinto, 2010) tanto en función del número de visitas a las distintas competencias del sitio web como de su grado relevancia e impacto en el conocimiento de los estudiantes participantes después de usar el portal. Las competencias mejores puntuadas son aprender a aprender, aprender a analizar, sintetizar y comunicar la información, aprender a crear conocimiento, y aprender a buscar y evaluar la información. La competencia peor valorada es la aprender a trabajar juntos. En relación con las subcompetencias, las mas interesantes para los estudiantes son las de aprender a analizar y aprender a aprender, mientras que la peor valorada es la de ética de la información. Se comprobó que el entrenamiento en el manejo del portal hizo que mejoraran las percepciones de los estudiantes para las categorías "aprender a escribir" y "aprender a trabajar en grupo", competencias cada vez más importantes para el desarrollo curricular de los estudiantes.

También se detectan algunas debilidades, cuya mejora requiere una mínima financiación para poder actualizar continuamente los contenidos y revisar los enlaces. En este sentido, hay algunas competencias, especialmente aquellas relacionadas con el uso de la tecnología, que por su emergencia y vertiginoso desarrollo tienen un ritmo de obsolescencia rápido y requieren un arduo esfuerzo por intentar mantenerlas.

\section{Conclusiones}

A partir del desarrollo y uso de estos portales de contenido sobre alfabetización informacional por parte de los distintos agentes de la comunidad universitaria, queremos resaltar cómo la alfabetización informacional se va integrando dentro del marco académico en el esfuerzo por desarrollar las competencias genéricas de los estudiantes universitarios, relacionadas con la búsqueda de información, la evaluación, el procesamiento, la difusión y comunicación, en un contexto marcado por la presencia de las nuevas tecnologías, el e-learning, la colaboración y el trabajo en equipo.

La experiencia ha demostrado que estos portales constituyen un recurso pedagógico para los procesos de enseñanza-aprendizaje, pues pro- 
mocionan el trabajo autónomo y el autoaprendizaje del estudiante, ya que pueden emplearse como ayuda y plataforma en clases presenciales, y también como recurso para la formación continua del estudiante, desde el autoaprendizaje; promocionan el trabajo en equipo del profesorado, contribuyendo tanto a los procesos formativos como a la cooperación interdisciplinaria; favorecen la innovación en metodologías de enseñanza-aprendizaje, contribuyendo a la actualización en metodologías de evaluación de conocimientos y competencias por parte de los estudiantes y suponen un escenario abierto con contenidos abiertos para que cualquier estudiante pueda adquirir las competencias y habilidades informacionales clave para la resolución de problemas tanto en su proceso formativo como en su quehacer profesional, al poder acceder a la información realmente útil y saber sacarle provecho. La capacidad de análisis, de síntesis y evaluación de la información será vital en el contexto del profesional del siglo XXI.

En resumen, los portales de contenido que hemos referenciado son una puerta abierta al conocimiento, dotados de contenidos de calidad y en formato abierto, traducido al lenguaje de los estudiantes y vehiculado a través de Internet con las herramientas de navegación, recuperación e interacción propias de la web y del elearning.

\section{Referencias}

American Library Association (1989). Presidential Committee on Information Literacy: Final Report. Chicago: American Library Association, 1989.

Altbach, P.; Reisberg, L.; Rumbley, L. (2009). Tras la pista de una revolución académica: Informe sobre las tendencias actuales. Paris: UNESCO, 2009

ANECA (2004). Libros blancos de los títulos de Grado. Madrid: ANECA, 2004. http://www.aneca.es/Documen tos-y-publicaciones/Libros-Blancos (2011-09-22)

Cvetkovic, B.V.; Lackie, R.J. (2009). Teaching Generation M: A Handbook for Librarians and Educators. New York: Neal-Schuman, 2009.

Comisión Europea (2003). El papel de las universidades en la Europa del conocimiento. Bruselas: Unión Europea. http://europa.eu.int/eur-lex/es/com/cnc/2003/com2003_0 058es01.pdf (2011-09-22)

Comisión Europea (2002). eEurope 2005: Una sociedad de la información para todos. Bruselas: Unión Europea. http://europa.eu.int/information society/eeurope/2002/ne ws_library/documents/eeurope2005/eeurope2005_es.pd f (2011-09-22)

Drucker, P. F. (1999). Managing Oneself. // Harvard Business Review. 77: 2 (1999) 65-74.

Eisler, D. (2000). The portal' progress: A gateway for access, information and learning communities. // Syllabus. 14:2 (2000) p.12-18

Garcia, J.; Pinto, M. (2010). Local versus global information relevance in Website use: a case study with the information literacy portal AlfinEEES. // Information Research. 15:4 (2010) 135-152.
Johnson, W. (2007). The Application of Learning Theory to Information Literacy. College \& Undergraduate Libraries. 14:4 (2007). 103-120.

Levy, P.; Roberts, S.R. (2005). Developing the New Learning Environment: The Changing Role of the Academic Librarian. London: Facet Publishing, 2005.

Konieczny, A. A. (2010). Experiences as an embedded librarian in online courses. Medical Reference Services Quarterly. 29:1 (2010) 47-57.

Ministerio de Ciencia e Innovación. Real Decreto 1393/2007 de 29 de octubre, por el que se establece la ordenación de las enseñanzas universitarias oficiales. Madrid: BOE, 2007.

Jafari, A. Educational Portal White Paper. Jafari, A.; Sheehan, M. (eds). Designing portals: opportunities and challenges. London: Information Science Publishing, 2003. 270-290.

Organisation for Economic Co-operation and Development (2005). The definition and selection of key competencies: Executive Summary, Organisation for Economic Co-operation and Development, Paris: OCDE, 2005. https://www.pisa.oecd.org/dataoecd/47/61/35070367.pdf (2011-09-22)

Pinto, M. (dir.) (2004). Portal e-COMS: Electronic content management skills (Tutorial hipertextual sobre alfabetización en información: habilidades para la gestión de los contenidos electrónicos). Universidad de Granada: Unidad de Innovación, 2004. http://www.mariapinto.es/ecoms (2011-09-22)

Pinto, M. (dir.) (2006). Alfin-EEES: Habilidades y competencias de gestión de la información para aprender a aprender en el marco del Espacio Europeo de Enseñanza Superior. Madrid: Ministerio de Educación, Ciencia y Cultura, 2006. http://www.mariapinto.es /alfineees/AlfinEEES.htm (2011-09-22)

Pinto, M.; Doucet, A. (2007). An Academic Portal for Higher Education Information Literacy: the e-COMS initiative. / Journal of Academic Librarianship. 33:5 (2007) 604-611.

Pinto, M.; Doucet, A. (2007). An Educational Resource for Information Literacy in Higher Education: Visibility and Usability of the e-COMS Academic Portal. // Scientometrics. 72: 2 (2007). 225-252.

Pinto, M.; Garcia, J. (2007). La enseñanza-aprendizaje de las competencias genéricas en el Espacio Europeo de la Educación Superior. // Innovación docente, tecnologías de la información y de la comunicación, e investigación educativa en la universidad de Zaragoza: caminando hacia Europa. Univesidad: Zaragoza, 2007.

Pinto, M.; Sales, D. (2007) A research case study for usercentred information literacy instruction: Information behaviour of translation trainees. // Journal of Information Science. 33: 5 (2007). 531-550.

Sales, D. (2008). Towards a student-centred approach to information literacy learning: A focus group study on the information behaviour of translation and interpreting students. // Journal of information literacy. 2:1 (2008). http://jil.lboro.ac.uk/ojs/index.php/JIL/article/view/RA-V211-2008-3 (2011-09-22)

Sharples, M.; Taylor, J.; Vavoula, G. (2005). Towards a theory of mobile learning. // mLearn. http://www.mlearn.org.za/CD/papers/Sharples.pdf

Xiao, J. (2010). Integrating information literacy into Blackboard: Librarian-faculty collaboration for successful student learning. // Library Management. 31: 8/9 (2010). 654-668.

York, A.C.; Vance, J.M. (2009). Taking Library Instruction into the Online Classroom: Best Practices for Embedded Librarians. // Journal of Library Administration. 49:1/2 (2009). 197-209. 
